Bezeichnung für alle Figuren.

No. 1. Vorderer, mit dem Wirbelkörper verwachsener Abschnitt.

No. 2. Hinterer Abschoitt als ein besonderer articulirender Knochen.

a Bogenbals

A Gelenkfortsatzsãule

$\left.\begin{array}{l}A^{\prime} \text { Vorderer } \\ A^{\prime \prime} \text { Hinterer }\end{array}\right\}$ Theil derselben

b Hinterer Querfortsatz

c Oberer Gelenkfortsatz

$\left.\begin{array}{l}c^{\prime} \text { Vorderer } \\ c^{\prime \prime} \text { Hinterer }\end{array}\right\}$ Theil desselben

d Unterer Gelenkfortsatz

e Zacke des Dornfortsatzes

$\left.\begin{array}{l}\text { a Vorderes } \\ \alpha^{\prime} \text { Hinteres }\end{array}\right\}$ Feld der Gelenkfläche des oberen Gelenkfortsatzes

$\beta$ Gelenkfläche des unteren Gelenkfortsatzes

$\gamma$ Gelenkfläche am vorderen Abschnitie zur Articulation mit dem

der zweibinteren, einen besonderen Knochen repräsentirenden Abschnitte

$\delta$ Gelenkfläche am vorderen Ende des hinteren Abschnittes zar Articulation mit dem vorderen

$\varepsilon$ Höcker an der hinteren Seite des unteren Gelenkfortsatzes

getheilten

Bogen-

hälfte.

\title{
III. (LX.) Ueber einige, durch einseitigen Defect des Bogenhalses am VI. Halswirbel bedingte Ibweichungen.
}

(Hierzu Taf. VIll. Fig. $2-4$. )

Vorgefunden vor mehreren Jahren an dem Skelete eines Weibes, dessen Wirbelsäule ich in meiner Sammlung aufbewahre.

\section{Halswirbel. (Fig, 2.)}

Der Wirbel ist etwas kleiner als der darüber liegende IV. Halswirbel. Er bat einen etwas niederen Körper, einen niederen Bogen, niedere GelenkTortsatzsäılen, namentlich einen schwachen, seitlich sehr comprimirten Dornfortsatz, welcher zwar etwas länger als der des IV. Halswirbels ist, aber von diesem an Dicke in verticaler Richtung um $\frac{1}{3}$, in transversaler Richtung um $\frac{2}{3}$ übertroffen wird. Dadurch und durch die versteckte Lage seiner rechtec Bogenhälfte fällt er vor allen andereo Halswirbeln auf.

Der Wirbel hat zwar im Ganzen die gewöbnliclse Form, aber sein Bogen ist, wegen des tieferen Abganges seines rechten Halses von dem Wirbelkörper, etwas schräg und so gestellt, dass dessen rechte Hälfte tiefer als die linke gelagert ist, und damit die höchste Stelle der rechten Gelenkfortsatzsäule $4 \mathrm{Mm}$. unter dern Niveau des oberen Handes des rechten leistenartigen Vorsprunges der oberen Endfläche des Wirbelkörpers steht, wăhrend die höchste Stelle der linken Gelenkfortsatzsäule zum Niveau des oberen Randes des leistenartigen Vorsprunges der entsprechenden Seite binaufgerückt sich vorfindet.

Archiv f. pathol. Anat. Bd.LXVIr. Hft. 3. 
Die rechte Bogenbälfte ist, abgesehen von ihrem tieferen Abgange vom Wirbelkörper, ausserdem noch mit folgenden Missstaltungen behaftet:

Der Hals dieser Bogerthälfte, welcher näher der unteren Endfläche des Wirbelbörpers abgeht, hat die Gestalt einer vierseitigen, in verticaler Richtung comprimirten Platte, welche die Flächen auf- und abwärts, die Ränder ein-rückwärts und vor-auswärts gerichtet hat, bis zur Gelenkfortsatzsäule 4,5 Mm. und in schräg sagittaler Richtung $7 \mathrm{Mm}$. breit, aussen und vorn ganz dünn, innen und hinten bis 2,5 $\mathrm{Mm}$. dick ist, während der Hals der linken Bogenbälfte, welcher der oberen Endfäche des Wirbelkörpers bis unter den leistenartigen Vorsprung desselben nahe gerückt ist, die Gestalt eines in schräg sagittaler Richtung etwas comprimirten Cylinders oder abgerundet vierseitigen Säule von etwas grösserer Länge, $5 \mathbf{M m}$. Dicke in verticaler und $4,5 \mathrm{Mm}$. in scbräg sagittaler Richtung anfweist.

Die Gelenkfortsatzsäule derselben Bogenbälfte (a) ist etwas deform, an der unteren Partie rückwärts sehr eingedrückt, hier an der Mitte tief eingeschnürt und nm 2-3 Mm. niederer als jene der linken Bogenhälfte. Sie weist allerdings zwei Gelenkfortsätze auf, aber der untere (a") derselben trägt nicht, wie derselbe Fortsatz der linken Seite und der der Norm, an der vorderen Seite, wo die Gelénkfläche feblt, sondern auf ganz anomale Weise und wie der obere Gelenkfortsatz, seine Gelenkfläche an der hinteren Seite $(\beta)$. Die Gelenkflächen beider Gelenkfortsätze liegen daher, nur durch eine quere raube Rinne $(\gamma)$ von $3 \mathrm{Mm}$. Breite, welche an der Einschnürung der Gelenkfortsatzsäule sich hefindet, von einander geschieden, unter einander. Die Gelenkflüche des oberen Gelenkfortsatzes $(\alpha)$ ist rhombisch, gross, anf- und rückwärts gekehrt und concav in transversaler Richtung; die Gelenkfläche des unteren Gelenkfortsatzes $(\beta)$ ist quer-oval, kleiner, steht fast vertical, ist aus- und rückwärts gerichtet und plan-convex.

VI. Halswirbel. (Fig. 3.)

Der Wirbel hat den Körper, den von ibm ausgehenden vorderen Querfortsatz der linken Seite, die linke Bogenhälfte mit der Gelenkfortsatzsäule und den ron da ausgehenden binteren oder eigentlichen Querfortsatz, die Knochenbrïcke zwischen beiden Querfortsälzen und das Foramen transversarium dieser Seite normal gebildet; übrigens zeigt er aber folgende Deformitäten:

Der rechte vordere Qnerfortsatz (a'), welcher vom vorderen Seitenwinkel des Wirbelkörpers ausgeht, ist kürzer als der der linken Seite, sonst aber normal angeordnet. Vom rechten hinteren Querfortsatze fehlt die vom Bogen sonst ausgehende Wurzel; es ist nur das jenseits des Foramen transversarium. befindliche Endstück (b') zugegen, welches von der die Querfortsätze verbindenden Knochenbrücke (c') getragen wird. Vom hinteren Seitenwinkel des Wirbelkörpers und an der Stelle, wo sonst der Bogenhals abgeht, setzt eine spiralförmig gedrehte, sehr schwache Spange (d'), die an ihrer Mitte 0,5 nnd $1 \mathrm{Mm}$. dick ist, zu dem nur mit selnem Endstïcke vorbandenen binteren Querfortsatz hinüber, wodurch das rechte Foramen transversarium ( $\mathrm{e}^{\prime}$ ) rückwärts geschlossen ist. Diese Spange ist der Rest des übrigens mangelnden Halses der rechten Bogenbälfte.

Der Bogen, welcher mit seiner rechten Hälfte etwas mebr rückwärts hervorsteht, als mit der linken, auch etwas tiefer gesenkt ist als diese, ist an seiner rechten Wurzel nicht geschlossen. 
Die rechte Bogenhälfte ist ohne Zusammenhang mit dem Wirbelkörper, weil, wie gesagt, ihr der Hals bis anf einen Rest, der zur Schliessung des Foramen transversarium verwendet ist, fehlt. Dieselbe ist auch ohne Zasammenhang mit dem vorbandenen Rudimente des hinteren Querfortsatzes. Die Lücke $\left(^{*}\right)$ an der Wurzel dieser Bogenbälfte, zwischen letzterer und dem Wirbelkörper, ist $7-8 \mathrm{Mm}$., die Lücke zwischen derselben und dem hinteren Querfortsatze $3 \mathrm{Mm}$. weit. Statt der gewöhnlichen Gelenkfortsatzsäule ist an ihr ein starker vor-, auf- und auswärts berrorragender, etwas hakenförmig nach abwärts gekrümmter Längshöcker ( $\left.f^{\prime}\right)$ zugegen, welcher eine obere innere, eine nntere vordere und eine hintere äussere Fläche aufweist. Die obere innere Fläche ist länglich vierseitig, plan. An ihrer vorderen Hälfte trägt sie eine ovale, schwach concave Gelenkfläche $(\alpha \alpha)$, welche $6 \mathrm{Mm}$. lang und $4 \mathrm{Mm}$. hoch ist. Die untere vordere rauhe Fläche ist lang und concav. Sie trägt $9 \mathrm{Mm}$. unter der oberen Fläche und $10-11 \mathrm{Mm}$. ein- nad rückwärts von der Spitze des Längshöckers, entsprechend der unteren Hälfte des Bogens, gleich über seipem unteren Rande, eine dreiseitige concave, $12 \mathrm{Mm}$. lange und $7 \mathrm{Mm}$. hohe Gelenkfläche. Die hintere äussere Fläche ist am oberen vorderen Theile sebr rauh, höckerig, gefurcht und sehr convex, am unteren hinteren Theile platt und etwas eingedrückt. Das ontere hintere Stïck $(\beta)$ des Längshöckers, mit der Gelenkfläche vorn, ist als der untere Gelenkfortsatz, das obere Stäck $(\alpha)$ mit einer kleinen Gelenkfläche $(\alpha \alpha)$ an seiner vorderen inneren Seite als der obere ganz anomale Gelenkfortsatz za nehmen.

Der Dornfortsatz hat die Charaktere desselben Fortsatzes am VII. Halswirbel.

Der Halstheil der Wirbelsäule ist etwas nach rechts gekrümmt.

Mangel des Halses der rechten Bogenhälfte des VI. Halswirbels bis auf eine das Foramen trausversarium rückwärts schliessende schwache Spange und Mangel der Wurzel des hinteren Querfortsatzes batten Trennung des Zusammenhanges der rechten Bogenhälfte mit dem Körper dieses Wirbels bewirkt. Diese Trennung zngleich mit Rückwärtstreten der rechten Eogenbälfte hatte die Bildung einer Lücke veranlasst, welche um $\frac{2}{7}$ weiter ist als der Abstand des leistenförmigen Vorsprunges am Wirbelkörper vom oberen Gelenkfortsatze an der linken Seite desselben Wirbels und etwas weiter oder doch nicht enger ist als der Abstand des unteren binteren Randes der rechten Gelenkfortsatzsäule vom Wirbelkörper am V. Halswirbel. Die untere Partie der rechten Gelenkfortsatzsäule am V. Halswirbel konnte daher die durch Defect entstandene rechtseitige Bogenlücke am VI. Halswirbel nicht überschreiten, um an die hintere Seite seiner rechtseitigen Gelenkfortsatzpartie zu gelangen. Die untere Partie der rechten Gelenkforsatzsäule des V. Halswirbels (Fig. 4 b), welche dem unteren Gelenkfortsatze der Norm entspricht, musste in der durch Defect entstandenen Lücke an der Wurzel der rech- 
ten Bogenhälfte des VI. Halswirbels (Fig. $4 \mathrm{c}$ ), vor dem oberen Theile des die Gelenkfortsatzportion repräsentirenden Längshöckers, welcher dem oberen Gelenkfortsatz der Norm entspricht, Platz nehmen, und damit auch diesseits der Gelenkfortsatzpartie die rechte Bogenhälfte des V. Halswirbels mit ihrem unteren Rande vor den oberen Rand der rechten Bogenbälfte des VI. Halswirbels gerückt werden. Es musste der untere rechte Gelenkfortsatz des V. Halswirbels, statt mit der vorderen, mit seiner hinteren Fläche mit dem unter ihr liegenden Wirbel, und der obere rechte Gelenkfortsatz des VI. Halswirbels, statt mit der hinteren, mit der vorderen Seite mit dem darüber liegenden Wirbel in Verbindung treten, d. j. an der rechten Seite der V. Halswirbel (Fig. 2) beide Gelenkforsäize ( $a^{\prime} a^{\prime \prime}$ ) mit hinteren Gelenkflächen $(\alpha, \beta)$, und an dem VI. Halswirbel (Fig. 3) beide Gelenkfortsätze $(\alpha, \beta)$ mit vorderen Gelenkflächen $(\alpha \alpha)$ verseben erhalten, der V. Halswirbel (Fig. 4 b) rückwärts nicht nur mit dem IV. (a), sondern auch anomaler Weise mit dem VI. Halswirbel (c) und der VI. Halswirbel vorwärts nicht nur mit dem VII. (d), sondern auch anomaler Weise mit dem V. Halswirbel (b) articuliren.

Ein äbnliches Beispiel einer durch Defect eines der Bogenhälse (Bogenwurzeln) bedingten anomalen Articulation beider GeJenkfortsätze, d. i. der Gelenkfortsätze in der Richtung. des Wirbels, welcher mit dem Defecte behaftet ist, mit dem darüber und darunter liegenden Wirbeln vorwärts und der Gelenkfortsätze des Wirbels, welcher̉ über dem an einem Defecte leidenden lagert, mit diesem Wirbel (unten) und dem darüber liegenden Wirbel (oben) rückwärts, ist, meines Wissens, noch nicht in der Literatur verzeichnet.

\section{Erklärung der Abbildungen.}

Taf. VIII. Fig. $2-4$.

Fig. 2. V. Halswirbel (Ansicht von hinten und rechts), a Rechte Gelenkfortsatzsäule. a' Oberer, a" unterer Gelenkfortsatz. a Gelenkfläche des oberen, $\beta$ des unteren Gelenkfortsatzes. $\gamma$ Raube Rinne zwischen beiden. b Linke Gelenkfortsatzsäule. b' Oberer, b" unterer Gelenkfortsatz.

Fig. 3. VI. Halswirbel (Ansicht von vorn und links). Linke Seite: a Vorderer, b hinterer Querfortsatz. c Knochenbrücke zwischen beiden. d Bogenbals. e Foramen transpersarium. f Gelenkfortsatzsäule. Rechte Seite: a' Vorderer Querfortsatz. b' Endstück des hioteren Querfortsatzes, c' Knochen- 
brücke zwischen beiden. $d^{\prime}$ Bogenhalsrest zur Schliessung des Foramed transversarium. e' Foramen transversarium. f' Längshöcker, welcher die Gelenkfortsatzsäule repräsentirt. $\alpha$ Partie, welche dem oberen, $\beta$ diejenige, welche dem unteren Gelenlfortsatze entspricht. $\alpha$ re Am oberen Gelenkfortsatze vorn statt hinten sitzende Gelenkfläcbe. $\left({ }^{*}\right)$ Lücke zwischen dem Körper und dem Bogen des Wirbels.

Fig. 4. Untere Partie der Halswirbelsäule zor Ansicbt der Wirbelbogen, abcd IV. bis VII. Halswirbel.

\section{IV. (LXI.) Erster Brustwirbel mit einem Foramen transversarium.}

Vorgefunden vor vielen Jahren an dem Skelete eines jungen männlichen Individuums, wovon ich die zur Erläuterung der Anomalie nöthigen Knochen in meiner Sammlung anf bewahre.

Alle Knochen des Skeletes, mit Ausnahme des I. Brustwirbels, verhielten sich normal.

Der I. Brustwirbel trägt an jeder Seite seines Körpers an und unter dem oberen Rande das Tuberculam costale mit einer ganzen Fossa costalis zur Articulation mit dem Capitulum der 1. Rippe und an dem unteren Rande das Segment eines ganz kleinen Tuberculum costale mit einem Theile der Fossa costalis zur Articulation mit dem Capitulum der 2. Rippe.

Der Wirbelbogen mit sejnen Theilen verhält sich, mit Ausnahme seines rechten Halses und seines rechten Querfortsatzes, ganz normal. Die Spitze jedes Querfortsatzes trägt an dem vorderen Umfange die Fossa transversalis zur Articulation mit dem Tuberculum der 1. Rippe jeder Seite.

Der rechte Wirbelbogenbals ist etwas kürzer und etwas schmäler (am $\frac{1}{9}$ ) als der linke. Der rechte Querfortsatz ist an seiner Basis in sagittaler Richtung etwas dicker als der linke. Der rechte Querfortsatz lässt $3,5 \mathrm{Mm}$. auswärts vom Bogenbalse von dem vorderen unteren Rande ein anomales, bakenförmig gekrümmtes Nebenfortsätzchen mit breiter Basis abgehen. Dieses bat die Gestalt einer horizontal liegenden, dreiseitigen Platte, welche mii dem abgestutzten Winkel schräg vor und einwärts zu einem anderen, ihm entgegen kommenden, ganz quer gestellten Fortsätzchen hervorsteht, das mit seinem Ende etwas einwärts gekrümmt erscheint. Dasselbe ist in verticaler Richtung comprimirt, weist zwei Flächen, zwei Ränder, eine Basis und eine Spitze auf. Seine Flächen kehrt es auf- und abwărts. Die obere Fläche ist in transversaler Richtung convex, in sagittaler Richtung concav, die untere Fläche ist plan. Von den Rändern ist der vordere äussere schwach S-förmig gekrümmt, der innere stark ausgebuchtet. Die Basis ist breit und liegt mit ihrer onteren Fläche in einer Ebene mit der Fläche des Querfortsatzes. Die Spitze ist abgestutzt. Seine Länge beträgt $5 \mathrm{Mm}$, seine Breite an der Basis $5 \mathrm{Mm}$., an der Spitze 1,25 Mm., seine Dicke $1,25-1,5 \mathrm{Mm}$.

Von der rechten Seite des Wirbelkörpers, zwischen dem oberen Theile des Bogenhalses und dem Tuberculum costale superius, steht quer und parallel der Bichtung des Querfortsatzes ein anderes, aber dreiseitig-pyramidales, am Ende ab- 